\title{
Detection of the Ammonium Ion in Space ${ }^{1}$
}

\author{
J. Cernicharo \& B. Tercero \\ Deparment of Astrophysics, CAB. INTA-CSIC. Crta Torrejón-Ajalvir Km 4. 28850 \\ Torrejón de Ardoz, Madrid, Spain \\ jcernicharo@cab.inta-csic.es \\ and \\ A. Fuente \\ Observatorio Astronómico Nacional, Apdo. 112, 28803, Alcalá de Henares, Spain \\ and \\ J.L. Domenech, M. Cueto, E. Carrasco, V.J. Herrero \& I. Tanarro \\ Instituto de Estructura de la Materia, IEM-CSIC, Serrano 123, 28006 Madrid, Spain \\ and \\ N. Marcelino \\ NRAO, 520 Edgemont Road, Charlottesville, VA 22902, USA \\ and \\ E. Roueff \\ Luth, Observatoire de Paris, CNRS UMR8102, Place J. Janssen F-92190 Meudon, France \\ and \\ M. Gerin \\ LERMA, Observatoire de Paris, CNRS UMR8112 and Ecole Normale Superieure, 61 \\ Avenue de lObservatoire, F-75014 Paris, France \\ and \\ J. Pearson \\ Jet Propulsion Laboratory, 4800 Oak Grove Drive, MC 168-314, Pasadena, CA 91109, \\ $U S A$
}




\begin{abstract}
We report on the detection of a narrow feature at $262816.73 \mathrm{MHz}$ towards Orion and the cold prestellar core B1-bS, that we attribute to the $1_{0}-0_{0}$ line of the deuterated Ammonium ion, $\mathrm{NH}_{3} \mathrm{D}^{+}$. The observations were performed with the IRAM $30 \mathrm{~m}$ radio telescope. The carrier has to be a light molecular species as it is the only feature detected over $3.6 \mathrm{GHz}$ of bandwidth. The hyperfine structure is not resolved indicating a very low value for the electric quadrupolar coupling constant of Nitrogen which is expected for $\mathrm{NH}_{3} \mathrm{D}^{+}$as the electric field over the $\mathrm{N}$ nucleus is practically zero. Moreover, the feature is right at the predicted frequency for the $1_{0^{-}} 0_{0}$ transition of the Ammonium ion, $262817 \pm 6$ $\mathrm{MHz}(3 \sigma)$, using rotational constants derived from new infrared data obtained in our laboratory in Madrid. The estimated column density is $(1.1 \pm 0.2) \times 10^{12}$ $\mathrm{cm}^{-2}$. Assuming a deuterium enhancement similar to that of $\mathrm{NH}_{2} \mathrm{D}$, we derive $N\left(\mathrm{NH}_{4}^{+}\right) \simeq 2.6 \times 10^{13} \mathrm{~cm}^{-2}$, i.e., an abundance for Ammonium of a few $10^{-11}$.
\end{abstract}

Subject headings: ISM: abundances — ISM: individual objects (B1-bS) - ISM: molecules — line: identification — molecular data

\title{
1. Introduction
}

Two of the major nitrogen bearing molecules in the ISM are $\mathrm{N}_{2}$ and $\mathrm{NH}_{3}$, which are predicted to be present in many different media (see, e.g., Nejad et al. 1990; Aikawa et al. 2008; Harada et al. 2010). Whereas $\mathrm{N}_{2}$ is expected to be largely in the gas phase, $\mathrm{NH}_{3}$ should be mostly frozen onto the surface of dust grains for temperatures lower than $100 \mathrm{~K}$. Diazenylium $\left(\mathrm{N}_{2} \mathrm{H}^{+}\right)$and Ammonium $\left(\mathrm{NH}_{4}^{+}\right)$, the protonated ions of $\mathrm{N}_{2}$ and $\mathrm{NH}_{3}$, can also provide crucial information for the understanding of interstellar chemistry. The $\mathrm{N}_{2} \mathrm{H}^{+}$ion is usually taken as a tracer for the apolar $\mathrm{N}_{2}$ molecule, which is practically impossible to observe directly using conventional spectroscopic techniques. Detection of diazenylium has been reported from many different sources (Turner 1974; Green et al. 1974; Fuente et al. 1993; Daniel et al. 2007). The ion is assumed to be formed almost exclusively through the reaction of $\mathrm{N}_{2}$ molecules with $\mathrm{H}_{3}^{+}$and to be destroyed mostly in collisions with electrons or with $\mathrm{CO}$ molecules. The existence of interstellar $\mathrm{NH}_{4}^{+}$is also predicted in various

\footnotetext{
${ }^{1}$ This work was based on observations carried out with the IRAM 30-meter telescope. IRAM is supported by INSU/CNRS (France), MPG (Germany) and IGN (Spain)
} 
astrochemical models. In particular, in hot cores (Aikawa et al. 2011) and in other high temperature environments like active galactic nuclei (Harada et al. 2010), grain evaporation leads to a large increase in the gas phase concentration of $\mathrm{NH}_{3}$. Ammonia has a high proton affinity and its protonated derivative, $\mathrm{NH}_{4}^{+}$, is predicted to become one of the dominant ions (Aikawa et al. 2008, 2011). Ammonium is also the dominant ion in many cold laboratory plasmas containing hydrogen and nitrogen (Carrasco et al. 2011, 2013). However, the symmetric character of $\mathrm{NH}_{4}^{+}$precludes its observation through radioastronomic methods, and $\mathrm{NH}_{4}^{+}$has never been detected in the ISM. Therefore, the deuterated variant $\mathrm{NH}_{3} \mathrm{D}^{+}$, possessing a small permanent dipole moment, is more suitable for astronomical searches.

Deuterated Ammonia $\mathrm{NH}_{2} \mathrm{D}$ was detected in Orion by Rodriguez-Kuiper et al. (1978) and in SgrB2 by Turner et al. (1978). A detailed analysis of the $\mathrm{NH}_{2} \mathrm{D} / \mathrm{NH}_{3}$ abundance ratio in Orion was first provided by Walmsley et al. (1987) who obtained a value of 0.003. Deuterated Ammonia is produced through the dissociative recombination of $\mathrm{NH}_{3} \mathrm{D}^{+}$with electrons. $\mathrm{NHD}_{2}$, and $\mathrm{ND}_{3}$ have been also detected towards a large variety of molecular clouds harbouring a large range of kinetic temperatures (Saito et al. 2000; Roueff et al. 2000; Lis et al. 2002; Roueff et al. 2005; Lis et al. 2006). Hence, the precursor molecule $\mathrm{NH}_{3} \mathrm{D}^{+}$, and even more deuterated isotopologues of the Ammonium ion, could be present in hot and cold molecular clouds.

In this Letter we report on the first detection in space of the singly deuterated Ammonium ion towards the sources Orion-IRc2 and the cold prestellar core B1-bS, a source where the whole family of ${ }^{15} \mathrm{~N}$ and deuterated isotopologues of Ammonia and $\mathrm{NNH}^{+}$have been detected (Lis et al. 2002, 2006; Saito et al. 2000; Daniel et al., in preparation).

\section{Observations}

The observations presented in this paper were motivated by the analysis of the Orion line survey carried out with the 30m IRAM telescope (Tercero et al. 2010, 2011). During the interpretation of the line survey we had to deal with more than 8000 unidentified features. Around 4400 of them have been successfully assigned to several isotopes of $\mathrm{CH}_{3} \mathrm{CH}_{2} \mathrm{CN}$, $\mathrm{CH}_{2} \mathrm{CHCN}, \mathrm{CH}_{3} \mathrm{OCOH}$, their vibrational levels and those of all abundant molecules, and to the recently detected methyl acetate and the gauche conformer of ethyl formate in this source

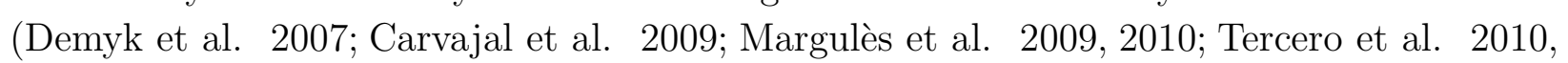
2011, 2012, 2013; Motiyenko et al. 2012; Daly et al. 2013; López et al. in preparation). Among the strongest unidentified features we found one at $262816.7 \mathrm{MHz}$ for $v_{L S R}=9 \mathrm{~km} \mathrm{~s}^{-1}$, (see Figure 1) that was very close to the predicted frequency for the $J_{K}=1_{0^{-}} 0_{0}$ line of $\mathrm{NH}_{3} \mathrm{D}^{+}$. This deuterated species of the Ammonium ion was observed in the laboratory 
by Nakanaga \& Amano (1986) through its $\nu_{4}$ vibrational mode from which rotational constants for the ground state were derived. The molecule was incorporated some time ago to the MADEX code (Cernicharo 2012). The calculated frequency for the $J_{K}=1_{0}-0_{0}$ transition of $\mathrm{NH}_{3} \mathrm{D}^{+}$from these rotational constants is $262807 \mathrm{MHz}$ with a $\pm 3 \sigma$ uncertainty of $\pm 9 \mathrm{MHz}$. The spectrum shown in Figure 1 shows a line $10 \mathrm{MHz}$ away from the predicted one with a linewidth similar to those of the $\mathrm{NH}_{2} \mathrm{D}$ lines observed in the same frequency survey. The observed feature, labelled U262816.7 in Figure 1, is practically free of blending from other species. None of the rotational transitions of the recently characterized isotopologues and vibrationally excited states of methyl/vinyl/ethyl cyanide, methyl formate, formamide, or from the abundant species found in Orion (Tercero et al. 2010) matches the frequency of U262816.7 except a possible very weak contribution from ${ }^{13} \mathrm{CH}_{3} \mathrm{OCOH}$ in its first torsional state. Nevertheless, having still 3600 unidentified features in Orion, claiming a possible identification with $\mathrm{NH}_{3} \mathrm{D}^{+}$will be extremely risky and speculative without a more precise frequency measurement in the laboratory (see Section 4).

Prompted by the observed line in Orion we started a search for a similar feature towards the cold prestellar cloud B1-bS. Complex organic molecules have been recently found in this cold core (Marcelino et al. 2009, 2010; Cernicharo et al. 2012). This source also shows an impressive enhancement in the deuteration of abundant molecules such as $\mathrm{H}_{2} \mathrm{CO}, \mathrm{H}_{2} \mathrm{CS}$, and $\mathrm{NH}_{3}$ (Lis et al. 2002, 2006; Marcelino et al. 2005; Saito et al. 2000). With a kinetic temperature of $\simeq 12 \mathrm{~K}$ the expected density of lines at $262.8 \mathrm{GHz}$ is rather low. Previous observations at 3 and 2 millimeter wavelengths indicate that only some diatomic and triatomic species will have significant emission at these high frequencies (Marcelino et al. 2005, 2009, 2010; Cernicharo et al. 2012).

The observations were performed at the IRAM 30m telescope at Pico Veleta (Spain) using the $1 \mathrm{~mm}$ Eight MIxer Receivers (EMIR). The backend was a fast Fourier Transform Spectrometer (FTS) with a spectral resolution of $50 \mathrm{kHz}$ and 37275 channels. Two bands, $1.82 \mathrm{GHz}$ wide each, were centered at 262.816 and $265.886 \mathrm{GHz}$. These two bands were observed simultaneously in the two orthogonal polarizations of the EMIR $230 \mathrm{GHz}$ receiver. All the observations were performed using the Wobbler Switching mode which produces remarkable flat baselines. Pointing errors were always within $3^{\prime \prime}$. The $30 \mathrm{~m}$ beam size at the observing frequency is $11^{\prime \prime}$. The spectra were calibrated in antenna temperature corrected for atmospheric attenuation using the ATM package (Cernicharo 1985; Pardo et al. 2001).

Two different runs have been used for the present observations. The first one in July 2012 allowed us to detect a feature at the same frequency of the line observed in Orion $(262816.73 \mathrm{MHz})$. During this run weather conditions were mostly average to moderate summer, with $3-10 \mathrm{~mm}$ of precipitable water vapor. System Receiver temperatures were 
between $80 \mathrm{~K}$ and system temperatures always above $400 \mathrm{~K}$. A second run in January 2013 provided much better weather conditions and sensitivities. The water vapour amount was 1-2 $\mathrm{mm}$ and system temperatures between 200-250 K. The final spectrum, with all data averaged, has a sensitivity of $4.9 \mathrm{mK}$ with $48.9 \mathrm{KHz}$ of spectral resolution $(3.7 \mathrm{mK}$ after a box smoothing of 2 channels). The total observing time was 50.8 hours with an averaged system temperature of $249 \mathrm{~K}$.

\section{Results}

The resulting spectrum from the $30 \mathrm{~m}$ telescope observations is shown in Figure 2. The upper panel shows the data at the original spectral resolution of $48.9 \mathrm{KHz}$. The lower panel shows the same data smoothed to $97.8 \mathrm{KHz}$. A feature is observed right at the expected frequency (see below) with a signal to noise ratio of 4.5 and 6 in the upper and lower panels, respectively. The line is narrow, having a width at half maximum of $0.65 \mathrm{~km} \mathrm{~s}^{-1}$. It appears as having a double peak that could be due to the hyperfine structure but it is within the noise of the data. Due to the central position of the nitrogen atom in Ammonium the electric field on the nucleus is close to zero. Hence, the quadrupolar coupling constant of the nitrogen nucleus is probably negligible. The Deuterium atom has a spin of 1 and could introduce also some hyperfine splitting but the corresponding quadrupolar momentum is expected to be also very low. The identification relies on the close match between the frequency of the feature with that predicted from the rotational constants derived with our new laboratory measurements (see below). Moreover, this is the unique feature detected together with the lines of $\mathrm{CCH} N=3-2$ over a bandwidth of $3.8 \mathrm{GHz}$. Taking into account the low kinetic temperature of the cloud no candidates can be found in MADEX, the CDMS, or JPL catalogues. Hence, the carrier has to be a light species and $\mathrm{NH}_{3} \mathrm{D}^{+}$is the best candidate. Note that in this source $\mathrm{NH}_{3},{ }^{15} \mathrm{NH}_{3}, \mathrm{NH}_{2} \mathrm{D},{ }^{15} \mathrm{NH}_{2} \mathrm{D}, \mathrm{ND}_{2} \mathrm{H}, \mathrm{ND}_{3}$ have been detected plus $\mathrm{NNH}^{+}, \mathrm{NND}^{+},{ }^{15} \mathrm{NNH}^{+}, \mathrm{N}^{15} \mathrm{NH}^{+}$(see above), so the chemistry is well constrained and the abundance of $\mathrm{NH}_{3}$ and its related species is high.

\section{New Laboratory Experiments}

The density of lines in B1-bS is low at these high frequencies and the agreement between the frequency of the observed feature with the predictions of Nakanaga \& Amano (1986) is very good. However, a final assignment relies on the precise determination from laboratory data of the rotational constants of $\mathrm{NH}_{3} \mathrm{D}^{+}$. We have measured again the $\nu_{4}$ band of deuterated Ammonium with better absolute frequency calibration and more lines than those 
reported by Nakanaga \& Amano (1986) $\left(2 \times 10^{-4}\right.$ vs. $1 \times 10^{-3} \mathrm{~cm}^{-1}$ and 76 vs. 61 lines respectively). A detailed description of these experiments and calculations are presented in the accompanying Letter (Doménech et al., 2013). The predicted frequency, calculated from the ground state rotational constants derived from a fit with the same parameters as those of Nakanaga \& Amano (1986) is $262817 \pm 6 \mathrm{MHz}( \pm 3 \sigma)$. The difference with the observed frequency is much less than one sigma deviation. Hence, we are fully confident that the detected feature in Orion and B1-bS corresponds to the $1_{0}-0_{0}$ transition of $\mathrm{NH}_{3} \mathrm{D}^{+}$.

\section{Gas phase chemical modeling}

The Orion data will be interpreted in a forthcoming paper. In this work we will concentrate on the analysis of the B1-bS data. Due to the low dipole moment of $\mathrm{NH}_{3} \mathrm{D}^{+}, \mu=0.26 \mathrm{D}$, the Einstein coefficient for spontaneous emission is $\mathrm{A}_{i j}=4.8 \times 10^{-6} \mathrm{~s}^{-1}$ and the critical density to efficiently pump the $1_{0}$ rotational level is just of a few $10^{4} \mathrm{~cm}^{-3}$. Hence, we could assume that the levels of $\mathrm{NH}_{3} \mathrm{D}^{+}$are thermalized at $T_{K}=12 \mathrm{~K}$ (Marcelino et al. 2005; Cernicharo 2012), we derive for the $A$ species of $\mathrm{NH}_{3} \mathrm{D}^{+}$a column density of $(5.5 \pm 1.0) \times 10^{11} \mathrm{~cm}^{-2}$. The total column density of deuterated Ammonium is, hence, $N\left(\mathrm{NH}_{3} \mathrm{D}^{+}\right)=(1.1 \pm 0.2) \times 10^{12} \mathrm{~cm}^{-2}$. The column density of molecular hydrogen derived by Hirano et al. (1999) is $1.3 \times 10^{23} \mathrm{~cm}^{-2}$. Therefore, the abundance of deuterated Ammonium is $\simeq 8 \times 10^{-12} . \mathrm{NH}_{2} \mathrm{D}$ has been observed by Saito et al. (2000) who obtained a column density of $(3.2 \pm 1.2) \times 10^{14} \mathrm{~cm}^{-2}$. Hence, the abundance ratio of deuterated Ammonia over the deuterated Ammonium ion is $\simeq 300$. Assuming a deuteration enhancement similar to that observed for $\mathrm{NH}_{2} \mathrm{D}$ (Saito et al. 2000) the abundance of Ammonium is $\simeq 1.610^{-10}$.

All deuterated isotopologues of Ammonia have been detected in B1-bS (Lis et al. 2002 , 2006; Saito et al. 2000) and the corresponding gas phase chemical models have been presented by Roueff et al. (2005) who were able to obtain satisfactory agreement between observations and gas phase models in which depletion effects of $\mathrm{C}$ and $\mathrm{O}$ on grains have been simulated. In these models, Ammonia formation results directly from dissociative recombination of the deuterated Ammonium ion which gives preferentially the $\mathrm{NH}_{3}+\mathrm{H}$ product (Öjekull et al. 2004). As a consequence, these models suggest that Ammonium is present at a significative level in this environment and its possible detection is a major witness of the occurrence of such a gas phase chemistry.

Nitrogen chemistry has received considerable recent interest (Dislaire et al. 2012) with the reevaluation of the starting step of gas phase chemical reactions leading to Ammonia via the Ammonium ion: $\mathrm{N}^{+}+\mathrm{H}_{2} \rightarrow \mathrm{NH}^{+}+\mathrm{H}$. This reaction is known to have a slight endothermicity and its efficiency is very much dependent on the para/ortho state of $\mathrm{H}_{2}$ 
i.e, wether rotational state of $\mathrm{H}_{2}$ is $0 / 1$, respectively. Indeed, based on low temperature experiments (Marquette et al., 1985), the endothermicity involved is $168.5 \mathrm{~K}$ with para- $\mathrm{H}_{2}$ and $41.9 \mathrm{~K}$ when $\mathrm{H}_{2}$ is in its "normal" form ( $1 / 4$ para $+3 / 4$ ortho). These values should be compared to the energy defect between the corresponding $J=0$ and $J=1$ levels of $\mathrm{H}_{2}$, which is $170.5 \mathrm{~K}$. Dislaire et al. (2012) have reevaluated the reaction rate constant involved with the ortho form of $\mathrm{H}_{2}$ with significant differences compared to the previous estimate of Le Bourlot (1991). An additional complexity, which is not yet fully understood, is the occurrence of fine structure of $\mathrm{N}^{+}$, whose levels are also split by similar amounts. These effects are neglected up to now.

Interestingly enough, thanks to the lower zero-point vibrational energy of $\mathrm{HD}$, the reactions

$$
\begin{aligned}
& \mathrm{N}^{+}+\mathrm{HD} \rightarrow \mathrm{ND}^{+}+\mathrm{H}, \\
& \mathrm{N}^{+}+\mathrm{HD} \rightarrow \mathrm{NH}^{+}+\mathrm{D}
\end{aligned}
$$

are found to have even smaller endothermicity. We derive a value of $16.3 \mathrm{~K}$ for the production of $\mathrm{ND}^{+}$and $100 \mathrm{~K}$ for the channel towards $\mathrm{NH}^{+}$. Once $\mathrm{NH}^{+}$or $\mathrm{ND}^{+}$are produced, they react with $\mathrm{H}_{2}, \mathrm{HD}, \mathrm{D}_{2}$ in a sequence of rapid exothermic reactions until formation of Ammonium. Walmsley and colleagues have been the first to include both para and ortho forms of $\mathrm{H}_{2}$, related ions $\left(\mathrm{H}_{2}^{+}, \mathrm{H}_{3}^{+}\right)$, and their multiply deuterated isotopologues for completely depleted regions (Walmsley et al. 2004; Flower et al. 2004, 2006a, b)), following an earlier suggestion of Pagani et al. (1992). We have extended this approach to a full chemical network including oxygen, carbon, sulfur and nitrogen (Pagani et al. 2011). For the purpose of the present paper, we have updated this chemical network with the recent findings on nitrogen chemistry reported above on the one hand and included also some recently determined neutral-neutral reaction rate constants (Daranlot et al. 2012). As a result, the present model contains 220 species (including ortho/para forms) of singly to fully deuterated substitutes and more than four thousand reactions. We display in Figure 3 the corresponding time evolution of the fractional abundances relative to molecular hydrogen of $\mathrm{NH}_{4}^{+}$and $\mathrm{NH}_{3} D^{+}$. The physical conditions correspond to those supposed to hold in B1-bS: the proton density is taken as $2 \times 10^{5} \mathrm{~cm}^{-3}$, a temperature $T=12 \mathrm{~K}$ is assumed, and the elemental abundances correspond to model 2 of Roueff et al. (2005). We see that the fractional abundances of $\mathrm{NH}_{4}^{+}$and $\mathrm{NH}_{3} \mathrm{D}^{+}$ peak are $\simeq 10^{-10}(0.7 \mathrm{Myr})$ and $\simeq 10^{-11}(1 \mathrm{Myr})$, respectively which is consistent with the observational derivations. The abundance ratio $X\left(\mathrm{NH}_{1}^{+}\right) / X\left(\mathrm{NH}_{3} \mathrm{D}^{+}\right)$is $\simeq 10$. This value is similar to that found for $\mathrm{NH}_{3} / \mathrm{NH}_{2} \mathrm{D}$ by Saito et al. (2000). For $\mathrm{t}=1 \mathrm{Myr}$ this ratio is $\simeq 2$.

Our chemical models predict that the abundance of $\mathrm{NH}_{2} \mathrm{D}_{2}^{+}$could be a factor of two lower than that of $\mathrm{NH}_{3} \mathrm{D}^{+}$. Consequently, we could expect to detect more deuterated species 
of the Ammonium ion in cold dark clouds. However, more accurate spectroscopic data are needed for $\mathrm{NH}_{2} \mathrm{D}_{2}^{+}$and $\mathrm{NHD}_{3}^{+}$in order to search for these species.

The Spanish authors thank the Spanish MICINN for funding support through grants, AYA2009-07304, CSD2009-00038, Fis2010-16455 and Fis2012-38175. M. Gerin and E. Roueff

acknowledge support from the french national PCMI program. J. Cernicharo thanks U. Paris Est for an invited professor position during complection of this work.

\section{REFERENCES}

Aikawa, Y., Wakelam, V., Garrod, R.T., \& Herbst, E., 2008, ApJ, 674, 993

Aikawa, Y., Furuya, V., Wakelam, F., et al., 2011, in IAU Symp. 280, The Molecular Universe, ed. J. Cernicharo and R. Bachiller, Cambridge University Press, 2011, p. 33

Carrasco, E., Jiménez-Redondo, M., Tanarro, I., \& Herrero, V. J., 2011, PCCP, 13, 19561

Carrasco, E., Tanarro, I., Herrero, V. J., \& Cernicharo, J., 2013, PCCP, 15, 1699

Carvajal, M., Margulès, L., Tercero, B., et al., 2009, A\&A, 500, 1109

Caselli, P., Benson, P. J., Myers, P. C., \& Tafalla, M., 2002, ApJ, 572, 238

Cernicharo, J., 1985, Internal IRAM report (Granada: IRAM)

Cernicharo, J., 2012, in ECLA-2011: Proceedings of the European Conference on Laboratory Astrophysics, EAS Publications Series, 2012, Editors: C. Stehl, C. Joblin, and L. d'Hendecourt (Cambridge: Cambridge Univ. Press), 251

Cernicharo, J., Marcelino, N., Roueff, E., et al., 2012, ApJ, 759, L43

Daniel, F., Cernicharo, J., Roueff, E., et al.,2007, M. L., ApJ, 667, 980

Daranlot, J., Hincelin, U., Bergeat, A., et al., 2012, PNAS, 109, 10233

Demyk, K., Mäder, H., \& Tercero, B., 2007, A\&A, 466, 255

Daly, A.M., Bermúdez, C., López, A., et al., 2013, ApJ, 768, 81

Domenech, J.L, 2013, Cueto, M., Herrero, V.J., et al., ApJ, accompanying Letter

Dislaire, V., Hily-Blant, P., Faure, A., et al., 2012, A\&A, 537, A20 
Flower, D.R., Pineau Des Forêts, G., \& Walmsley, C.M., 2004, A\&A, 427, 887

Flower, D.R., Pineau Des Forêts, G., \& Walmsley, C.M., 2006a, A\&A, 449, 621

Flower, D.R., Pineau Des Forêts, G., \& Walmsley, C.M., 2006b, A\&A, 456, 215

Fuente, A., Martin-Pintado, J., Cernicharo, J., \& Bachiller, R., 1993, A\&A, 276, 473

Green, S., Montgomery, J.A. Jr., \& Thaddeus, P., 1974, ApJ, 193, L89

Harada, N., Herbst, E., \& Wakelam, V., 2010, ApJ, 721, 1570

Hirano, N., Kamazaki, T., Mikami, H., et al., 1999, in Star Formation 1999, ed. T. Nakamoto (Nagano: NRO), 181

Le Bourlot, J., 1991, A\&A, 242, 235

Lis, D.C., Roueff, E., Gerin, M., et al., 2002, ApJ, 571, L55

Lis, D.C., Gerin, M., Roueff, E., et al., 2006, ApJ, 636, 916

Marcelino, N., Cernicharo, J., Roueff, E., et al., 2005, ApJ, 620, 308

Marcelino, N., Cernicharo, J., Tercero B., \& Roueff, E., 2009, ApJ, 690, L27

Marcelino, N., Brünken, S., Cernicharo, J., et al., 2010, A\&A, 516, A105

Marquette, J.B., Rowe, B.R., Dupeyrat, G., \& Roueff, E., 1985, A\&A, 147, 115

Margulès, L., Motiyenko, R.A., Demyk, K., et al., 2009, A\&A, 493, 565

Margulès, L., Huet, T.R., Demaison, J., et al., ApJ, 714, 1120

Motiyenko, R.A., Tercero B., Cernicharo, J., \& Margulès, L., 2012, A\&A, 548, A71

Nakanaga, T., \& Amano, T., 1986, Can. J. Phys., 64, 1356

Nejad, L. A. M., Williams, D. A., \& Charnley, S. B., 1990, MNRAS, 246, 183

Öjekull, J., Andersson, P.U., Na ${ }^{\circ} \mathrm{Ga}^{\circ}$ Rd, M. B., et al., J. Chem. Phys., 120, 7391

Pagani, L., Salez, M., \& Wannier, P.G., 1992, A\&A, 258, 479

Pagani, L., Roueff, E., \& Lesaffre, P., 2011, ApJ, 739, L35

Pardo, J. R., Cernicharo, J., \& Serabyn, E. 2001, IEEE Trans. Antennas and Propagation, $49 / 12,1683$ 
Rodriguez-Kuiper, E.N., Zuckerman, B., \& Kuiper, T.B.H., 1978, ApJ, 219, L49

Roueff, E., Tiné, S., Coudert, L.H., et al., 2000, A\&A, 354, L63

Roueff, E., Lis, D.C., van der Tak, F.F.S., et al., 2005, A\&A, 438, 585

Saito, S., Ozeki, H., Ohishi, M., \& Yamamoto, S., ApJ, 535, 227

Tercero, B., Cernicharo, J., Pardo, J. R., \& Goicoechea, J. R., 2010, A\&A, 517, 96

Tercero, B., Vincent, L., Cernicharo, J., et al., 2011, A\&A, 528, 26

Tercero, B., Margulès, L., Carvajal, M., et al., 2012, A\&A, 538, A119

Tercero, B., Kleiner, I., Cernicharo, J., et al., 2013, ApJ, 770, L13

Turner, B.E., 1974, ApJ, 193, L83

Turner, B.E., Zuckerman, B., Morris, M., \& Palmer, P., 1978, ApJ, 219, L43

Walmsley, C.M., Hermsen, W., Henkel, C., et al., 1987, A\&A, 172, 311

Walmsley, C.M., Flower, D.R., \& Pineau des Forêts, G., 2004, A\&A, 418, 1035 


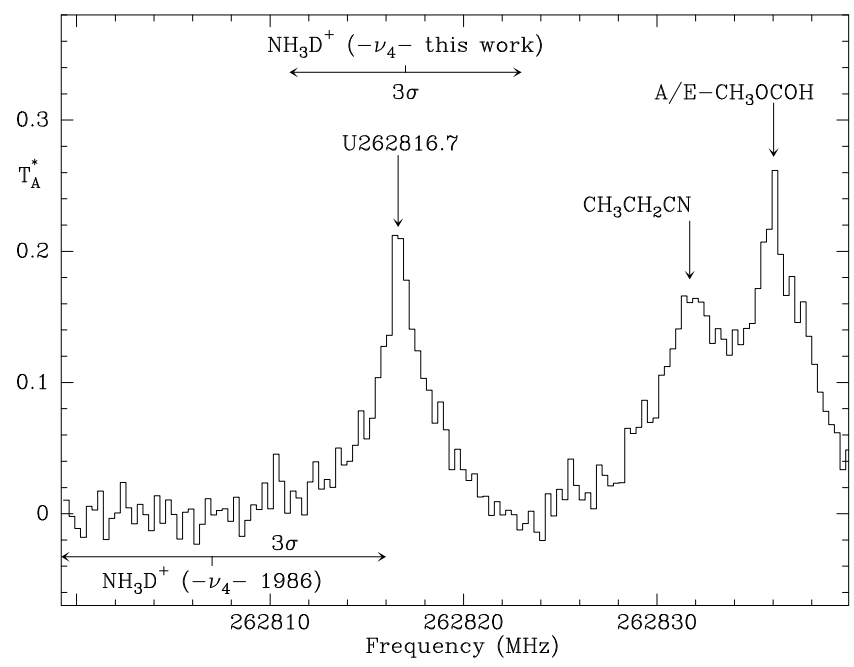

Fig. 1.- Observed spectrum towards Orion-IRc2 around the expected frequency of the $J_{K}=1_{0^{-}} 0_{0}$ line of the deuterated ammonium ion. Identification of all other lines in this frequency range is indicated. The predicted frequency and its $3 \sigma$ error bar is indicated at the bottom for the Nakanaga \& Amano (1986) measurements and at the top for our new laboratory data (Domenech et al., 2013; see accompanying Letter). 


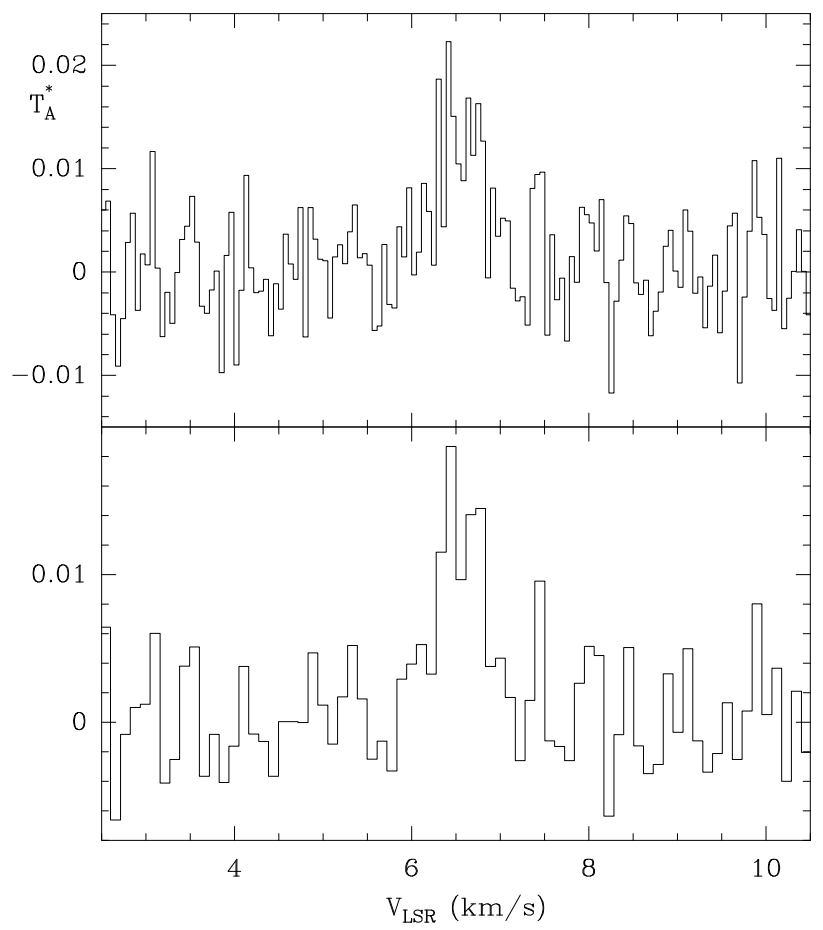

Fig. 2.- Observed spectrum towards B1-bS $\left(\alpha_{2000}=03^{h} 33^{m} 21.34^{s}, \delta_{2000}=31^{\circ} 07^{\prime} 26.7^{\prime \prime}\right)$ at the expected frequency of $\mathrm{NH}_{3} \mathrm{D}^{+} J_{K}=1_{0^{-}} 0_{0}$ transition. The upper panel contains the raw data resulting from a total integration time of 51 hours with a spectral resolution of 48.8 $\mathrm{KHz}\left(0.054 \mathrm{~km} \mathrm{~s}^{-1}\right)$. The bottom panel shows the same data smoothed to $98 \mathrm{KHz}$. For a LSR velocity of the source of $6.5 \mathrm{~km} \mathrm{~s}^{-1}$ the derived line frequency for the observed feature is $262816.73 \pm 0.1 \mathrm{MHz}$. 


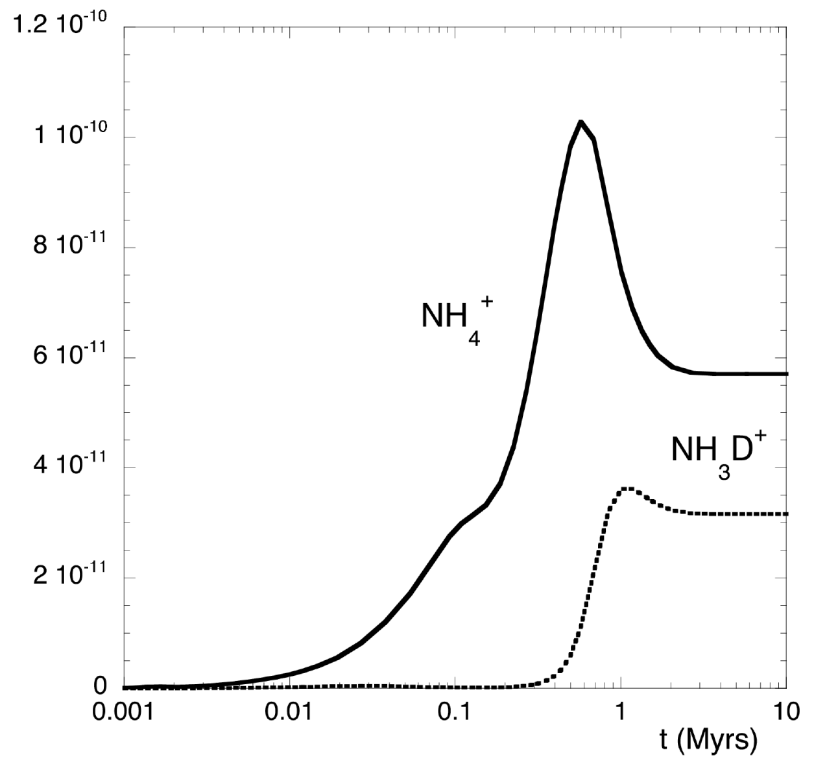

Fig. 3.- Abundances of $\mathrm{NH}_{4}^{+}$and $\mathrm{NH}_{3} \mathrm{D}^{+}$as a function of time for the chemical model described in the text. 